\title{
Desventajas competitivas en la producción y provisión de los mercados de flores de Santa Rosa y Piedra Liza
}

Edgard Venegas Palacios* JOHNNY ÁLVAREZ OCHOA**

\section{RESUMEN}

El manejo de la producción de las flores tiene algunas deficiencias y en la comercialización no existe una distribución adecuada. En este trabajo se realizará un estudio a partir de la provisión de flores a los mercados de Santa Rosa y Piedra Liza donde se concentra la comercialización de este producto en Lima, a partir del mismo se estudiará la subyacencia de la respectiva cadena productiva. Los resultados permitirán mostrar recomendaciones para mejorar las condiciones en la producción y comercialización.

Palabras clave: cadena productiva, comercialización, potencial económico, producción de flores

COMPETITIVE DISAdVANTAGES IN PROdUCING AND providing to Santa Rosa and Piedra Liza FLOWERS MARKETS

\section{ABSTRACT}

Managing the production of flowers has some shortcomings in marketing and there is no suitable distribution. In this work, a study was conducted from providing flowers to Santa Rosa and Piedra Liza markets, which the commercialization of this product is concentrated in Lima, from the same subjacency the respective supply chain is studied. The results will show recommendations for improving the production and marketing.

Keywords: economical potential, flower production, productive chain, marketing

\section{INTRODUCCIÓN}

El interés de desarrollar este trabajo de Clúster del mercado de flores fue principalmente porque el Perú posee una variedad muy rica de flores (follajes exóticos de la selva, flores de desierto, flores de verano que se producen en la sierra) y sus exportaciones a Estados Unidos están libres del pago de arancel gracias al Tratado de Libre Comercio (TLC) bilateral, por lo que se muestra como un fuerte competidor en este mercado.

La importancia del presente trabajo radica en que actualmente, Estados Unidos es el principal destino de las flores peruanas con el $65 \%$ de lo exportado, seguido de Países Bajos con el $24 \%$, y el resto distribuido entre Italia, Chile, Canadá y otros [1].

Asimismo, el Perú ocupa el noveno lugar como proveedor de flores al mercado estadounidense, con exportaciones de US\$ 5.8 millones en el 2012, monto que significó un crecimiento de más de $15 \%$ respecto al 2011 [2].

A pesar de las limitaciones y deficiencias en la producción y comercialización, el clúster de flores peruano, tiene potencial para seguir desarrollándose y obtener una ventaja competitiva por medio de TICs y su efecto en el fortalecimiento del modelo de Porter [3], partiendo de un módulo de planificación que aunque es una actividad de apoyo, está relacionado con la producción del cultivo. El objetivo es sacar provecho del vínculo entre actividades integrando la información que generan:

- Logística de Entrada: la integración vertical con proveedores mejora la eficiencia en los procesos.

- Producción: un módulo de planificación y monitoreo contribuiría a su organización y efectividad.

- Comercialización y Ventas: información adicional recolectada en post-cosecha sería útil para que Ventas no ofrezca productos que no tiene.

- Servicio al cliente: información consolida del cliente estrecharía las relaciones y serviría para ofrecerle servicios de valor.

El estudio será realizado mediante entrevistas a los comerciantes de los mercados de flores de Santa Rosa y Piedra Liza, que

\footnotetext{
* Mag, estudiante de doctorado y docente de la Universidad Nacional de Ingeniería. E-mail: kenny@imca.edu.pe

** Mag, estudiante de doctorado en la UNI y Jefe de la Of. De Presupuesto del Pliego del Ministerio
} de Trabajo y Promoción del Empleo. E-mail: johnnyalvarez025@gmail.com 
es enfocado como el componente territorial y de proximidad geográfica del cluster, donde una parte de la cadena también subyace. Ya el análisis de la cadena es complementado considerando no solamente el componente subyacente, si no la cadena en su totalidad.

\section{CLUSTERS EN EL PERÚ E INNOVACIÓN}

Álvarez [1] sostiene que "en los cluster encontramos un conocimiento compartido por lo general en un determinado sector. Este conocimiento compartido podría ser tácito o codificado". El autor presenta, en la tabla, la siguiente identificación de clusters en el Perú:

\begin{tabular}{|l|l|l|}
\hline \multicolumn{1}{|c|}{ Denominación } & \multicolumn{1}{c|}{ Sector } & \multicolumn{1}{c|}{ Área geográfica } \\
\hline "Gamarra" & Confecciones & Distrito de la Victoria \\
\hline "Wilson" & Software y diseño gráfico & cercado de Lima \\
\hline Vitivinícola de Ica & Vitivinícola & Región Ica \\
\hline Pte. Sta. Rosa & Arreglos florales & Distrito del Rímac \\
\hline Atem & Metalmecánica & Distrito de Los Olivos \\
\hline "Caquetá" & Cuero y calzado & Rímac-San Martin \\
\hline "El Porvenir" & Calzados & Ciudad de Trujillo \\
\hline Muebles de madera & Muebles de madera & Villa El Salvador \\
\hline Minero del sur & Minería & Toquepala - Cuajone \\
\hline
\end{tabular}

Por otro lado, D'Avila [5] ha realizado un estudio, por encargo del Consejo Nacional de la Competitividad y el Ministerio de la Producción, que ha consistido en el mapeamiento de clusters en el Perú en el cual se muestra que la Asociación de Mercado de Flores de Santa Rosa y Piedra Liza no se ha considerado como cluster debido a la inexistencia de una masa crítica relevante y significativa. Según Jean Guinet [6] "los clusters económicos surge con mayor frecuencia cuando hay una masa crítica de empresas que permitan establecer economías de escala y alcance, una sólida base científica y tecnológica, y una cultura favorable a la innovación y el espíritu empresarial", lo cual sería la fundamentación de lo mostrado en el informe de D'Avila.

A pesar de que Guinet afirma también que "las agrupaciones también pueden basarse en factores tales como los recursos naturales o ventajas geográficas. Muchos grupos exitosos tienen raíces históricas de largo, y el surgimiento de nuevos grupos lleva tiempo", no determinaremos si estamos frente a un cluster o a una organización sectorial [7].

Sin embargo, podemos mencionar que en estos mercados se carece de la promoción de la innovación, de lo contrario existiría una mayor capacidad para percibir nuevas necesidades de los clientes y nuevas posibilidades tecnológicas, comerciales o productivas mediante la investigación.
Este estudio no revela la presencia de una capacidad de innovación necesaria para sostener una posición competitiva en comparación de mercados de flores en Ecuador y Colombia, además la calidad de rosas y claveles producidas en estos países son superiores.

\section{RELACIÓN ENTRE LOS COMERCIANTES Y LOS GOBIERNOS LOCALES}

La floricultura [8] en Maracás está intensamente relacionada con el gobierno local, quien ha sido el responsable de un crecimiento de representación en esta actividad, en comparación con escenario antes de que la presencia del Estado. Comparando la realidad de Maracás y la de Lima, podemos observar que el crecimiento en la producción y comercialización de flores no se debe a la Municipalidad de Lima, pues a pesar de sus esfuerzos en organizar a los comerciantes de los mercados de Santa Rosa y Piedra Liza no se ha logrado un avance significativo. Recordemos que estos comerciantes han sido reubicados de la Explanada sur del Estadio Nacional hace muchos años y luego de 18 años recién cuentan con un mercado techado y piso de cemento pulido.

Hay que notar que la falta de organización de los directivos de los mercados de flores no ha permitido gestionar facilidades para el 
ordenamiento y la comercialización, ellos mismos reconocen que tienen iniciativa para mejorar sus ambientes y han presentado un proyecto para contar con un estacionamiento y que los mercados tengan una infraestructura con dos niveles, el cual fue rechazado. Además han solicitado a las municipalidades del Rímac y Lima un mejor servicio para el recojo de la basura, pues ya han sido multados en enero del 2013 con 1, 850 nuevos soles por el arrojo de basura en la calle.

En la actualidad la cantidad de desperdicios arrojados en la vía pública proviene principalmente de los comerciantes que se encuentran ubicados a los alrededores de los mercados, quienes en su mayoría no están organizados y además perjudican a la comercialización, debido a que los precios en relación a lo establecido dentro de los mercados son mayores. Cuando el precio es menor se debe a que la calidad de las flores disminuye pues se ofrece los productos que no llegan en óptimo estado debido al transporte.

\section{VENTAJAS Y DESVENTAJAS EN EL SISTEMA DE COMERCIALIZACIÓN}

\subsection{Ventajas}

- Abastecen clientes del mercado local: florerías, comerciantes de mercados y consumidores finales, donde los dos primeros clientes además de la calidad de flores, buscan un abastecimiento permanente, el cumplimiento de compromisos (servicio) y homogeneidad de los productos.

- Es posible una integración vertical en la cadena productiva, con la implementación de florerías y la aplicación de la mano de obra familiar especialmente de los jóvenes (hijos de los productores) quienes pueden incursionar en el negocio de la industrialización de las flores a través de la fabricación de arreglos florales, lágrimas y coronas de caridad, generando empleo.

- La producción local de flores puede desplazar las importaciones efectuadas actualmente por las florerías, mayoristas y minoristas que operan en el mercado local buscando minimizar los costos de transporte.

- La principal ventaja es aprovechar favorablemente los factores climáticos de los lugares de donde provienen las flores a nivel regional. Los precios se incrementan en el mercado local debido a que la oferta local se reduce a su mínima expresión e ingresa producción extra regional e internacional. Las principales desventajas de estos respecto a la producción local son los mayores costos de flete a los centros de consumo.

- Mirar el mercado local como la gran alternativa, considerando aspectos tan relevantes como la tecnología de producción, material vegetal de origen y la organización para manejar la postcosecha de las flores, tema este último en el que existe un desconocimiento casi total. La asesoría técnica a la que acceden generalmente es deficiente y escasamente calificada.

\subsection{Desventajas}

- Baja cotización de las flores producidas localmente, debido a su baja calidad, especialmente en las rosas y claveles, que no presentan el tamaño, grosor de tallo y diámetro de flor requerido para competir con las flores importadas, así mismo no tienen buena presentación.

- La fijación de precios es por parte del comerciante, especialmente en la época de abundancia de oferta de flores y porque los productores no están debidamente organizados para la comercialización.

- El transporte inadecuado de las flores afectan aún más la calidad de las flores ya en desventaja frente a las importadas debido a la inexistencia de un tratamiento adecuado en el proceso de post cosecha.

- No utilizan técnicas de tratamiento que prolonguen la resistencia de las plantas y su periodo de percibilidad en el proceso de distribución y comercialización.

- Falta desarrollar los productos: logo, empaques, etiquetas, información sobre la calidad, tratamiento, usos, recomendaciones al usuario final para su adecuado mantenimiento.

- Abastecimiento de flores sin mayor tratamiento y/o acondicionamiento y/o diversificación de la presentación de sus productos los pone en desventaja frente a los importados y le determina bajos precios y bajos niveles de negociación.

- Falta de mecanismos y estrategias técnicas que optimicen el adecuado transporte y manejo de post-cosecha como el uso de cajas, no permiten en general, llegar al consumidor con una flor de buena calidad, lo que se traduce también en una disminución al precio pagado al productor.

- Comercialización individual y desorganizada que también incide en su capacidad de negociación en el mercado regional. 
- Los productores no gestionan empresarialmente la producción de flores, no llevan registros, ni estiman sus costos de producción y desconocen los márgenes de utilidad que estos les genera.

\section{CONCLUSIONES Y RECOMENDACIONES}

\subsection{En el sistema técnico productivo}

En lo técnico productivo, se evidencia un patrón de Tecnología tradicional de uso actual que no aporta en la mejora de la productividad, calidad y competitividad de las especies obtenidas. Van acompañados de escasos o nulos niveles de manejo de información técnica, emergencia o desarrollo de experiencias de propuestas alternativas de diversificación sobre la base de ensayos o experiencias cuantificables, evaluación de la adaptación y comportamiento de diferentes materiales productivos a las condiciones ambientales locales.

Los productores no cuentan con el apoyo de instituciones gubernamentales y no gubernamentales en el desarrollo de investigación aplicada que pueda mejorar su competitividad.

Su estructura productiva es también elemental, no ha desarrollado suficientemente el uso de invernaderos y otras alternativas, principalmente por los costos y por la incompatibilidad con el funcionamiento de la estructura de trabajo familiar. Para el proceso de la post no cuentan con instalaciones adecuadas limitando la posibilidad de manejar mejor el periodo de perecibilidad y su calidad.

\subsection{En el sistema de comercialización}

Los canales de distribución están poco organizados, se evidencia un mal manejo del producto en el proceso de comercialización, debido al desconocimiento técnico y falta de instalaciones apropiadas para el manejo en el proceso de post cosecha y comercialización de las flores por parte de los productores y agentes intervinientes en el sistema (distribuidores mayoristas y minoristas). Generando una gran merma de flores y una pérdida de calidad del producto, que se aprecia a simple vista en la marchites de las flores y perdida de belleza, las flores y hojas dañadas. Se estima que este problema afecta hasta el $30 \%$ de la producción que es verificado por el mayorista quien procede a castigar en el precio, afecta también en el periodo de vida de la flor en el florero poniéndolas en desventaja frente a las especies importadas que duran hasta 15 días.
Esta situación incide en los elevados márgenes de comercialización que se traducen en precios altos al consumidor final, se estima que estos se incrementan hasta $1100 \%$ veces sobre el precio que recibe el productor, que le implican costos/ beneficios no mayores al $26 \%$.

\subsection{En el sistema organizacional}

Los productores no se han organizado para la producción ni comercialización lo que no les permite generar ahorros 0 abaratar costos en los procesos de abastecimiento de materiales, insumos, tecnología, capacitación etc. Así como en el proceso de transporte y comercialización de la producción, que les permitiría ampliar su margen de beneficio o reducción de sus pérdidas.

La falta de estrategias de gestión organizativa productiva, comercial y financiera, inciden en los bajos niveles de competitividad y eficiencia individual y colectiva, por lo que se deben desarrollar estas capacidades que fortalezcan la comercialización organizada para posicionarse en el mercado de flores a nivel regional y ampliar progresivamente en el mediano plazo su cobertura a nivel nacional e internacional.

\section{RECOMENDACIONES}

Tomar en cuenta los criterios determinantes para lograr competitividad en el mercado, para lograr una mayor valoración se deben presentar las Flores frescas, limpias, sin daño alguno, con buena forma y color, con uniforme grado de maduración, tamaño y rigidez, libres de plagas y enfermedades, sin desviaciones en su crecimiento, con tallos largos con la longitud y rigidez correcta.

El empaque es necesario para transportar las plantas por lo que debe optimizarse de tal forma que minimice los costos de transporte. Un buen empaque protege a las flores contra daños causados por el calor, transporte y manipulación, además de que debe ser parte del desarrollo del producto.

El producto se debe comercializar en envases reciclables de dimensiones adecuadas para trasladar las flores, incluyendo papel picado y sujetadores, para prevenir daños en el proceso de transporte, ya que los productos son muy sensibles. En el empaque debe referirse información sobre la calidad, el origen, cuidados y manipulación que sirva al comerciante y consumidor final y evitar pérdidas por inadecuados tratamientos. 


\section{REFERENCIAS BIBLIOGRÁFICAS}

[1] Álvarez, J.C (2010) "Nuevos espacios productivos: Parques tecnológicos vs. clusters empresariales" en Memorias del II Congreso Peruano de Investigación de Operaciones Copios 2010. Arequipa, Perú.

[2] D’Avila J. (2013) "Elaboración de un mapeo de clusters en el Perú". Consultoría solicitada por el Consejo Nacional de la Competitividad y el Ministerio de la Producción. Lima, Perú.

[3] Chaminade C., Vang, J. (2006), "Innovation policy for Asian SMEs: exploring cluster differences". DRUID Summer Conference 2006, June 18-20. Copenhagen, Denmark.

[4] Herrera A., Giraldo O., Rueda T., Arias R. (2003)."Competitividad del Cluster Floricultor de la Sabana de Bogotá", Trabajo para el curso "Firmas, Cluster y Competitividad". Bogotá, Colombia.

[5] Vivanco C., Álvarez, J.C., Vodden, K. (2011). "Extracción de algas en Pisco: Desafíos, oportunidades, adaptación y perspectivas futuras”. Industrial Data. Vol. 14, N 1: 19-27.
[6] Lira, J. (2014). Perú es el noveno proveedor de flores al mercado estadounidense. http:// gestion.pe/economia/peru-noveno-proveedorflores-al-mercado-estadounidense-2085962, (visitado el 03-08-14).

[7] Vizcaíno D., Jaramillo, A., Zúñiga R. (2001). "Diagnóstico de la Agrotecnología en Ecuador". Instituto Centroamericano de Administración de Empresas. Ecuador.

[8] Porter M. (1998). "On Competition”, Ed. Boston: Harvard Business School Press.

[9] Passos, E.,(2010). "O arranjo produtivo de flores em maracás (BA) sob o enfoque da inovação". $X X X$ encontro nacional de engenharia de produção. São Carlos, SP, Brasil.

[10]Mixteo, J., Idigoras, I., Vicente, A. (2003). "Los clusters como fuente de competitividad: el caso de la Comunidad Autónoma del País Vasco". Universidad del País Vasco/Euskal Herriko Unibertsitatea.

[11]Guinet, J. (1999). "Introduction". Boosting Innovation: The Cluster Approach. OECD proceedings. 